趴 


\section{Populationsbiologie der Pflanzen}

Herausgegeben von Bernhard Schmid und Jürg Stöcklin

\section{Springer Basel AG}


Anschrift der Herausgeber:

Dr. Bernhard Schmid

Dr. Jürg Stöcklin

Botanisches Institut der Universität Basel

Schönbeinstraße 6

CH-4056 Basel

\section{CIP-Titelaufnahme der deutschen Bibliothek}

Populationsbiologie der Pflanzen / hrsg. von Bernhard Schmid und Jürg Stöcklin. - Basel ; Boston ; Berlin : Birkhäuser, 1991

NE: Schmid, Bernhard [Hrsg.]

ISBN 978-3-0348-5638-6 ISBN 978-3-0348-5637-9 (eBook)

DOI 10.1007/978-3-0348-5637-9

Das Werk ist urheberechtlich geschützt. Die dadurch begründeten Rechte, insbesondere die der Übersetzung, des Nachdruckes, der Entnahme von Abbildungen, der Funksendung, der Wiedergabe auf photomechanischem oder ähnlichem Weg und der Speicherung in Datenverarbeitungsanlagen bleiben, auch bei nur auszugsweiser Verwertung, vorbehalten. Die Vergütungsansprüche des $\S 54$, Abs. 2 UrhG werden durch die «Verwertungsgesellschaft Wort», München, wahrgenommen.

(C)1991 Springer Basel AG

Ursprünglich erschienen bei Birkhäuser Verlag, P. O. Box 133,CH-4010 Basel, Switzerland 1991.

Printed from the authors' camera-ready manuscripts on acid-free paper 


\section{INHALT}

Vorwort

R. BORNKAMM, A. EGGERT, M. KÜPPERS, B. SCHMID, J. STÖCKLIN

Liste populationsbiologisch relevanter Begriffe

P. POSCHLOD

1) Diasporenbanken in Böden - Grundlagen und Bedeutung 15

M. JENNY

2) Diasporenausbreitung an ariden Standorten und ihre Klassifikation am Beispiel einer Annuellenflur im Wadi Araba (Jordanien) ........36

J. J. SCHNELLER

3) Besiedelungsstrategie und Populationsentwicklung am Beispiel des Farns Asplenium ruta-muraria

W. EBER UND B. VEENHUIS

4) Natalität und Mortalität bei Limonium vulgare 62

E.-G. MAHN UND L. UDWAL

5) Zur phänotypischen Plastizität bei Populationen von Chenopodium album $\mathrm{L}$.

R. CORNELIUS UND D. HAUG

6) Zur Plastizität des reproduktiven Aufwandes bei Conyza canadensis und Tanacetum vulgare

P. FAVRE UND J. STŌCKLIN

7) Regulation der Reproduktion bei Epilobium dodonaei Vill. 96

D. MATTHIES

8) Räumliche und zeitliche Dynamik in Populationen der seltenen Art Melampyrum arvense L.

A. H. MEYER UND B. SCHMID

9) Experimentelle Demographie von Pflanzen: Solidago altissima 123 


\section{J. STÖCKLIN UND H. ZOLLER}

10) Vergleich von Lebenszyklus und Populationsstruktur bei Höhenvikarianten der Gattung Epilobium.

M. KÜPPERS

11) Die Bedeutung des Wechselspiels von Photosynthese, Blattpopulation und pflanzlicher Architektur für Wachstum und Konkurrenzkraft.

P. EGLI UND B. SCHMID

12) Beziehung zwischen Struktur und Dynamik von Blattpopulationen und Sprosswachstum

B. SCHMID

13) Konkurrenz bei Pflanzen

P. STOLL, J. WEINER UND B. SCHMID

14) Grōssenvariabilität in einer Population von Pinus silvestris 211

W. SEIDLING

15) Verteilungsmuster der Pflanzen am Waldboden und deren Ursachen

T. TSCHARNTKE

16) Die Auswirkungen der Herbivorie auf Wachstum und Konkurrenzfähigkeit von Pflanzen

H. MŨLLER

17) Pflanzenreaktionen auf Wurzelherbivoren - Variationen, Ursachen und Mechanismen: Experimente an zweijährigen

Kompositen

R. CORNELIUS

18) Zur Bedeutung populationsbiologischer Forschung für den speziellen Naturschutz

Literaturverzeichnis

Index 


\section{VORWORT}

Das Projekt für dieses Buch entstand im Mai 1990 anlässlich des jährlichen Treffens des populationsbiologischen Arbeitskreises der Gesellschaft für Ökologie (GfÖ). Die Autoren möchten damit den gegenwärtigen Stand der populationsbiologischen Forschung im deutschsprachigen Raum dokumentieren und einen Überblick über das Spektrum dieser Forschungsrichtung geben.

Die mitteleuropäische Pflanzenökologie hat ihre Wurzeln in der naturgeschichtlichen Tradition und war deshalb lange geprägt von einer mehr qualitativen, beschreibenden Betrachtungsweise. Die Dringlichkeit vieler Umweltprobleme erfordert zunehmend, dass der Einfluss lokaler und globaler Umweltveränderungen oder die Folgen spezifischer Eingriffe auf die Vegetation vorausgesagt werden können. Dazu ist eine quantitative, analytische Betrachtungsweise notwendig. Der populationsbiologische Ansatz berücksichtigt, dass Vegetation aus Individuen besteht, die bestimmte Beziehungen zueinander und zur unbelebten Umwelt aufweisen. Die Populationsbiologie ermöglicht deshalb die Integration über mehrere, meist getrennt behandelte, hierarchische Stufen Organismus - Population - Lebensgemeinschaft - Ökosystem und kann eine Verbindung zwischen den traditionellen Disziplinen der Ökophysiologie und der Vegetationskunde herstellen.

Die Populationsbiologie fragt nach der Anzahl und Grösse von Pflanzen, welche an einem Ort gefunden werden können und nach der Art und Weise, wie sich die Populationsgrösse verändert. Sie fragt nach der Alters- oder Grössenverteilung der Pflanzen und dem Schicksal von Samen, Keimlingen, genetischen Individuen oder Pflanzenteilen. Das Umschlagsbild dieses Buches zeigt die Verteilung von Föhren (kreisförmige Symbole) und einigen anderen Bäumen in einem Wald. Weil Pflanzen im Gegensatz zu vielen Tieren sesshaft sind und kontinuierlich wachsen, sind die Position und die Grösse von Individuen besonders wichtige Variablen. Obwohl das Bild nur eine vorgefundene Situation wiedergibt, regt es unmittelbar zu vielen ökologisch relevanten Fragen an: Lässt sich ein räumliches Muster erkennen? Kann etwas über die Heterogenität des Standortes oder die Besiedlungsgeschichte ausgesagt werden? oder: Welche Nachbarbeziehungen haben die Bäume zueinander? Das Beispiel veranschaulicht unser wichtigstes Ziel, die Leser für eine neue ökologische Arbeits- und Betrachtungsweise zu motivieren, welche unvoreingenommen Informationen über Pflanzen in ihrer natürlichen Umgebung präzise darstellt und durch die Interpretation dieser 
Beobachtungen zu einem kausalen Verständnis von Vegetationsstruktur und Vegetationsentwicklung beiträgt.

Die Kapitel des Buches umfassen fünf thematische Schwerpunkte. Der erste und längste Teil (Kap. 1-10), welcher die Demographie und Dynamik von Pflanzenpopulationen beschreibt, ist entsprechend dem Ablauf des Lebenszyklus von Pflanzen gegliedert. Kapitel 1 und 2 beschreiben die Biologie von Diasporenpopulationen, Kapitel 3 beschreibt die Besiedlungsstrategie und Populationsgründung einer Farnpflanze und Kapitel 4 das Wachstum einer langlebigen, bereits etablierten Rhizompflanze. Die Kapitel 5-7 konzentrieren sich auf die populationsbiologische Bedeutung der Reproduktion und Diasporenproduktion. In den Kapiteln 8-10 schliesslich werden exemplarisch der Lebenszyklus und die Populationsdynamik einer seltenen annuellen Art, eines Neophyten und von Pionierpflanzen beschrieben. Im zweiten Teil (Kap. 11-12) werden nicht Populationen ganzer Pflanzen, sondern Populationen von Blättern beschrieben und ihre ökophysiologische Bedeutung für die Architektur der ganzen Pflanze und die Bestandesstruktur untersucht. Der dritte Teil des Buches (Kap. 13 - 15) behandelt Konkurrenzphänomene, Grössenvariabilität und räumliche Muster in Pflanzenpopulationen, und im vierten Teil (Kap. 16 - 17) werden Beziehungen zwischen Pflanzen und ihren Frassfeinden beschrieben. Das abschliessende Kapitel 18 schliesslich ist der Bedeutung populationsbiologischer Fragestellungen für den Natur- und Artenschutz gewidmet.

Erfahrene Leser werden sich fragen, warum dieses Buch nicht in englischer Sprache veröffentlicht wurde. Wir haben ganz bewusst für deutschsprachige Leser geschrieben, um den Studierenden der Biologie eine junge, vorwiegend in Grossbritannien und Nordamerika etablierte Disziplin näherzubringen und zu demonstrieren, dass die Populationsbiologie von Pflanzen auch in unseren Sprachraum Eingang gefunden hat. Es ist keineswegs unsere Absicht, eine 'kontinentale' Populationsbiologie der Pflanzen zu propagieren, vielmehr möchten wir dieser Disziplin insgesamt zu noch mehr Verbreitung verhelfen. Wir sind aber überzeugt, dass der reiche Erfahrungshintergrund ökologischer Disziplinen in Mitteleuropa die Populationsbiologie um neue Gesichtspunkte zu bereichern vermag und möchten hoffen, dass dieses Buch dazu beiträgt.

An dieser Stelle möchten wir allen danken, die uns Arbeiten eingesandt haben oder solche begutachtet haben. Zu ganz besonderem Dank sind wir allen verpflichtet, die mitgeholfen haben, die Manuskripte inklusive die Figuren, Tabellen und Literaturangaben in eine endgültige Form zu bringen: P. Egli, J.-N. Haas, A.H. Meyer, H. Schneider. 